\title{
THE BAILEY TRANSFORM AND FALSE THETA FUNCTIONS
}

\author{
GEORGE E. ANDREWS ${ }^{1}$ AND S. OLE WARNAAR ${ }^{2}$
}

\begin{abstract}
An empirical exploration of five of Ramanujan's intriguing false theta function identities leads to unexpected instances of Bailey's transform which, in turn, lead to many new identities for both false and partial theta functions.
\end{abstract}

\section{INTRODUCTION}

On page 13 of Ramanujan's Lost Notebook 9] (c.f. [4, Section 9.3, pp. 227-232], we find the following five identities:

$$
\begin{gathered}
\sum_{n=0}^{\infty} \frac{(-1)^{n} q^{n(n+1)}\left(q ; q^{2}\right)_{n}}{(-q ; q)_{2 n+1}}=\sum_{n=0}^{\infty}(-1)^{n} q^{n(n+1) / 2} \\
\sum_{n=0}^{\infty} \frac{\left(q ; q^{2}\right)_{n}^{2} q^{n}}{(-q ; q)_{2 n+1}}=\sum_{n=0}^{\infty}(-1)^{n} q^{n(n+1)} \\
\sum_{n=0}^{\infty} \frac{\left(q ; q^{2}\right)_{n} q^{n}}{(-q ; q)_{2 n+1}}=\sum_{n=0}^{\infty}(-1)^{n} q^{3 n(n+1) / 2} \\
\sum_{n=0}^{\infty} \frac{(q ;-q)_{2 n} q^{n}}{(-q ; q)_{2 n+1}}=\sum_{n=0}^{\infty}(-1)^{n} q^{2 n(n+1)} \\
\sum_{n=0}^{\infty} \frac{(q ;-q)_{n}\left(-q^{2} ; q^{2}\right)_{n} q^{n}}{(-q ; q)_{2 n+1}}=\sum_{n=0}^{\infty}(-1)^{n} q^{3 n(n+1)}
\end{gathered}
$$

where $(A ; q)_{n}=(1-A)(1-A q) \cdots\left(1-A q^{n-1}\right)$ and $|q|<1$.

From the right-hand sides of these identities we see that each is an instance of the false theta series (c.f. [10, $\S 9]$ )

$$
\sum_{n=0}^{\infty}(-1)^{n} q^{n(n+1) / 2}
$$

with $q$ replaced respectively by $q, q^{2}, q^{3}, q^{4}$ and $q^{6}$. However, this regularity is completely obscured in the left-hand sides.

2000 Mathematics Subject Classification. 33D15, 11P83.

Key words and phrases. Ramanujan, false theta series, Bailey transform, Bailey pair.

${ }^{1}$ Partially supported by National Science Foundation Grant DMS 0457003.

${ }^{2}$ Supported by the Australian Research Council. 
On the other hand, each of the five left-hand sides is of the form

$$
\sum_{n=0}^{\infty} \frac{t_{1} t_{2} \cdots t_{n} q^{n}}{(-q ; q)_{2 n+1}}
$$

and one naturally asks: Did Ramanujan find all the instances of 1.3 in which the resulting power series is a false theta series (not necessarily an instance of $(1.2)$ )?

A computer algebra search using MACSYMA revealed the instances $z=1,-1$, $\mathrm{i}, \mathrm{e}^{\pi \mathrm{i} / 3}$, and $\mathrm{e}^{2 \pi \mathrm{i} / 3}$ of the following identity which apparently escaped Ramanujan's attention:

$$
\begin{aligned}
\sum_{n=0}^{\infty} \frac{\left(-z q ; q^{2}\right)_{n}\left(-z^{-1} q ; q^{2}\right)_{n} q^{n}}{(-q ; q)_{2 n+1}} & =\sum_{n=0}^{\infty} \frac{1-z^{2 n+1}}{1-z} z^{-n} q^{n(n+1)} \\
& =\sum_{n=0}^{\infty} \frac{1+z+z^{2}+\cdots+z^{2 n}}{z^{n}} q^{n(n+1)} .
\end{aligned}
$$

Once (1.4) has been discovered, the next questions are: (1) How do you prove it, and (2) Where does it fit in the classical theory of $q$-series?

In Section 2, we lay the ground work for answers to these questions by considering instances of a bilateral version of the classic Bailey Transform. In Section 3, we derive four identities that will yield a number of applications of the results in Section 2 to false and partial theta functions. In Section 4 we prove (1.1a) $1.1 \mathrm{~d}$, and in Section 5 we prove a number of related results including the Lost Notebook entry [4, p. 239, Entry 9.5.3]

$$
\sum_{n=0}^{\infty} \frac{q^{n}}{\left(-q ; q^{2}\right)_{n+1}}=\sum_{n=0}^{\infty}(-1)^{n} q^{2 n(3 n+2)}\left(1+q^{4 n+2}\right),
$$

the elegant

$$
\sum_{n=1}^{\infty} \frac{\left(-q^{2} ; q^{2}\right)_{n-1} q^{n}}{\left(-q ; q^{2}\right)_{n}\left(1+q^{2 n}\right)}=\sum_{n=1}^{\infty} n q^{n^{2}}
$$

and the rather curious

$$
\sum_{n=0}^{\infty} \frac{\left(-q^{4} ; q^{4}\right)_{n-1} q^{n}}{\left(-q^{2} ; q^{2}\right)_{n-1}\left(-q ; q^{2}\right)_{n+1}}=1+\sum_{n=1}^{\infty}(-1)^{f(n)} q^{2 n}
$$

where

$$
f(n)=n+k \quad \text { if } \quad 2 k^{2}-2 k+1 \leq n \leq 2 k^{2}+2 k .
$$

We conclude with a few comments about unexplored aspects of the Bailey Transform.

\section{The Bilateral Bailey Transforms}

In [5, p. 1], W. N. Bailey first presented the basic result which has become known as Bailey's Transform [16, Section 2.4, pp. 58-74]. If

$$
\beta_{n}=\sum_{r=0}^{n} \alpha_{r} u_{n-r} v_{n+r} \quad \text { and } \quad \gamma_{n}=\sum_{r=n}^{\infty} \delta_{r} u_{r-n} v_{r+n},
$$

then

$$
\sum_{n=0}^{\infty} \alpha_{n} \gamma_{n}=\sum_{n=0}^{\infty} \beta_{n} \delta_{n}
$$


subject to conditions on the four sequences $\alpha_{n}, \beta_{n}, \gamma_{n}$ and $\delta_{n}$ which make all the infinite series absolutely convergent.

For our purposes we require two bilateral versions of Bailey's Transform.

Lemma 2.1 (Symmetric Bilateral Bailey Transform). If

$$
\beta_{n}=\sum_{r=-n}^{n} \alpha_{r} u_{n-r} v_{n+r} \quad \text { and } \quad \gamma_{n}=\sum_{r=|n|}^{\infty} \delta_{r} u_{r-n} v_{r+n}
$$

then

$$
\sum_{n=-\infty}^{\infty} \alpha_{n} \gamma_{n}=\sum_{n=0}^{\infty} \beta_{n} \delta_{n}
$$

subject to conditions on the four sequences $\alpha_{n}, \beta_{n}, \gamma_{n}$ and $\delta_{n}$ which make all the relevant infinite series absolutely convergent.

Proof.

$$
\begin{aligned}
\sum_{n=0}^{\infty} \beta_{n} \delta_{n} & =\sum_{n=0}^{\infty} \delta_{n} \sum_{r=-n}^{n} \alpha_{r} u_{n-r} v_{n+r} \\
& =\sum_{r=-\infty}^{\infty} \alpha_{r} \sum_{n=|r|}^{\infty} \delta_{n} u_{n-r} v_{n+r} \\
& =\sum_{r=-\infty}^{\infty} \alpha_{r} \gamma_{r} .
\end{aligned}
$$

Lemma 2.2 (Asymmetric Bilateral Bailey Transform). Let $m=\max \{n,-n-1\}$. If

$$
\beta_{n}=\sum_{r=-n-1}^{n} \alpha_{r} u_{n-r} v_{n+r+1} \quad \text { and } \quad \gamma_{n}=\sum_{r=m}^{\infty} \delta_{r} u_{r-n} v_{r+n+1}
$$

then

$$
\sum_{n=-\infty}^{\infty} \alpha_{n} \gamma_{n}=\sum_{n=0}^{\infty} \beta_{n} \delta_{n}
$$

subject to conditions on the four sequences $\alpha_{n}, \beta_{n}, \gamma_{n}$ and $\delta_{n}$ which make all the relevant infinite series absolutely convergent.

Proof. See the proof of Lemma2.1.

In the remainder of the paper we refer to pairs of sequences $\left(\alpha_{n}, \beta_{n}\right)$ satisfying (2.1) or 2.3) as Bailey pairs, dropping the adjectives (a)symmetric and bilateral. Similarly, we refer to pairs $\left(\gamma_{n}, \delta_{n}\right)$ satisfying (2.1) or 2.3) as conjugate Bailey pairs.

\section{Conjugate Bailey pairs}

We shall obtain four conjugate Bailey pairs $\left(\gamma_{n}, \delta_{n}\right)$. The first two are related to the Symmetric Bilateral Bailey Transforms and the last two to the Asymmetric Transform. 
Theorem 1. If we take $u_{n}=v_{n}=1 /\left(q^{2} ; q^{2}\right)_{n}$ and

$$
\delta_{n}=\frac{\left(q^{2} ; q^{2}\right)_{2 n} q^{n}}{(-q ; q)_{2 n+1}}
$$

in the Symmetric Bilateral Bailey Transform, then

$$
\gamma_{n}=q^{|n|} \sum_{j=0}^{\infty} q^{j^{2}+(2|n|+1) j}=q^{-n^{2}} \sum_{r=|n|}^{\infty} q^{r(r+1)} .
$$

Proof. Let $m=|n|$. After substituting (3.1) as well as $u_{n}=v_{n}=1 /\left(q^{2} ; q^{2}\right)_{n}$ in (2.1) we obtain the identity

$$
\sum_{r=m}^{\infty} \frac{\left(q^{2} ; q^{2}\right)_{2 r} q^{r}}{(-q ; q)_{2 r+1}\left(q^{2} ; q^{2}\right)_{r-m}\left(q^{2} ; q^{2}\right)_{r+m}}=q^{-m^{2}} \sum_{r=m}^{\infty} q^{r(r+1)}
$$

To prove this we begin by shifting the summation index $r$ to $r+m$ on both sides. Using $(A ; q)_{n+k}=(A ; q)_{k}\left(A q^{k} ; q\right)_{n}$ this leads to

$$
\sum_{r=0}^{\infty} \frac{\left(q^{4 m+2} ; q^{2}\right)_{2 r} q^{r}}{\left(-q^{2 m+2} ; q\right)_{2 r}\left(q^{2} ; q^{2}\right)_{r}\left(q^{4 m+2} ; q^{2}\right)_{r}}=(-q ; q)_{2 m+1} \sum_{r=0}^{\infty} q^{r(r+2 m+1)} .
$$

Instead of proving this we will establish the more general

$$
\sum_{r=0}^{\infty} \frac{\left(a^{2} ; q^{2}\right)_{2 r} q^{r}}{(-a q ; q)_{2 r}\left(q^{2} ; q^{2}\right)_{r}\left(a^{2} ; q^{2}\right)_{r}}=\frac{(-q ; q)_{\infty}}{(-a q ; q)_{\infty}} \sum_{r=0}^{\infty} a^{r} q^{r^{2}}
$$

from which 3.2 follows by setting $a=q^{2 m+1}$.

Using $(A ; q)_{2 n}=\left(A ; q^{2}\right)_{n}\left(A q ; q^{2}\right)_{n}$ and $\left(A^{2} ; q^{2}\right)_{n}=(A ; q)_{n}(-A ; q)_{n}$, and employing standard $q$-hypergeometric notation, 3.3 may also be stated as

$$
{ }_{3} \phi_{2}\left(\begin{array}{l}
a,-a, a q \\
a^{2},-a q^{2}
\end{array} q^{2}, q\right)=\frac{(-q ; q)_{\infty}}{(-a q ; q)_{\infty}} \sum_{r=0}^{\infty} a^{r} q^{r^{2}} .
$$

By virtue of the transformation [8, p. 100; Exercise 3.2]

$$
{ }_{3} \phi_{2}\left(\begin{array}{c}
a,-a, b \\
a^{2}, b z
\end{array} ; q,-z\right)=\frac{(z ; q)_{\infty}}{(b z ; q)_{\infty}}{ }_{2} \phi_{1}\left(\begin{array}{c}
b, b q \\
a^{2} q
\end{array} q^{2}, z^{2}\right), \quad|z|<1
$$

this simplifies to

$$
{ }_{2} \phi_{1}\left(\begin{array}{c}
a q, a q^{3} \\
a^{2} q^{2}
\end{array} ; q^{4}, q^{2}\right)=\frac{\left(-q^{2} ; q^{2}\right)_{\infty}}{\left(-a q ; q^{2}\right)_{\infty}} \sum_{r=0}^{\infty} a^{r} q^{r^{2}}
$$

For later reference we will replace $a \rightarrow a / q$ and then rescale $q^{2}$ to $q$, viz.,

$$
{ }_{2} \phi_{1}\left(\begin{array}{c}
a, a q \\
a^{2}
\end{array} ; q^{2}, q\right)=\frac{(-q ; q)_{\infty}}{(-a ; q)_{\infty}} \sum_{r=0}^{\infty} a^{r} q^{\left(\begin{array}{c}
r \\
2
\end{array}\right)} .
$$

By Heine's transformation [8, p. 359, Equation (III.2)]

$$
{ }_{2} \phi_{1}\left(\begin{array}{c}
a, b \\
c
\end{array} ; q, z\right)=\frac{(c / b ; q)_{\infty}(b z ; q)_{\infty}}{(c ; q)_{\infty}(z ; q)_{\infty}}{ }_{2} \phi_{1}\left(\begin{array}{c}
a b z / c, b \\
b z
\end{array} ;, \frac{c}{b}\right)
$$

(3.4) further simplifies to

$$
\sum_{r=0}^{\infty} \frac{\left(a ; q^{2}\right)_{r}}{\left(a q ; q^{2}\right)_{r}} a^{r}=\sum_{r=0}^{\infty} a^{r} q^{\left(\begin{array}{c}
r \\
2
\end{array}\right)}, \quad|a|<1 .
$$


Since

$$
\sum_{r=0}^{\infty} a^{r} q^{\left(\begin{array}{c}
r \\
2
\end{array}\right)}=\sum_{n=0}^{\infty} a^{2 n} q^{n(2 n-1)}\left(1+a q^{2 n}\right)
$$

this may be recognized as a special instance of the Rogers-Fine identity [7, Equation $(14.1)]$

$$
\sum_{n=0}^{\infty} \frac{(a ; q)_{n}}{(b ; q)_{n}} z^{n}=\sum_{n=0}^{\infty} \frac{(a ; q)_{n}(a z q / b ; q)_{n}}{(b ; q)_{n}(z ; q)_{n+1}}\left(1-a z q^{2 n}\right)(b z)^{n} q^{n(n-1)} .
$$

Theorem 2. If we take $u_{n}=v_{n}=1 /\left(q^{2} ; q^{2}\right)_{n}$ and

$$
\delta_{n}=(q ; q)_{2 n} q^{n}
$$

in the Symmetric Bilateral Bailey Transform, then

$$
\gamma_{n}=\sum_{r=0}^{\infty} q^{\left(\begin{array}{c}
r+1 \\
2
\end{array}\right)+(2 r+1)|n|}=q^{-2 n^{2}} \sum_{r=2|n|} q^{\left(\begin{array}{c}
r+1 \\
2
\end{array}\right)} .
$$

Proof. Let $m=|n|$. After substituting (3.5) as well as $u_{n}=v_{n}=1 /\left(q^{2} ; q^{2}\right)_{n}$ in 2.1 we are to prove the identity

$$
\sum_{r=m}^{\infty} \frac{(q ; q)_{2 r} q^{r}}{\left(q^{2} ; q^{2}\right)_{r-m}\left(q^{2} ; q^{2}\right)_{r+m}}=q^{-2 m^{2}} \sum_{r=2 m}^{\infty} q^{\left(\begin{array}{c}
r+1 \\
2
\end{array}\right)} .
$$

On the left we shift $r \rightarrow r+m$ and on the right $r \rightarrow r+2 m$. Hence

$$
\sum_{r=0}^{\infty} \frac{\left(q^{2 m+1} ; q\right)_{2 r} q^{r}}{\left(q^{2} ; q^{2}\right)_{r}\left(q^{4 m+2} ; q^{2}\right)_{r}}=(-q ; q)_{2 m} \sum_{r=0}^{\infty} q^{\left(\begin{array}{r}
r+1 \\
2
\end{array}\right)+2 r m} .
$$

This may readily be recognized as (3.4) with $a=q^{2 m+1}$.

The next two theorems claim conjugate Bailey pairs corresponding to the Asymmetric Bilateral Bailey transform.

Theorem 3. If we take $u_{n}=v_{n}=1 /\left(q^{2} ; q^{2}\right)_{n}$ and

$$
\delta_{n}=\frac{\left(q^{2} ; q^{2}\right)_{2 n+1} q^{n}}{(-q ; q)_{2 n+2}}
$$

in the Asymmetric Bilateral Bailey Transform, then

$$
\gamma_{n}=\sum_{r=0}^{\infty} q^{r(r+2)+(2 r+1) m}=q^{-n(n+1)} \sum_{r=m}^{\infty} q^{r(r+2)},
$$

where $m=\max \{n,-n-1\}$.

Proof. First we observe that by $u_{n}=v_{n}$ and $m=\max \{n,-n-1\}$ we have

$$
\gamma_{n}=\sum_{r=m}^{\infty} \delta_{r} u_{r-n} u_{r+n+1}=\sum_{r=m}^{\infty} \delta_{r} u_{r-m} u_{r+m+1} .
$$

Substituting (3.6) and $u_{n}=1 /\left(q^{2} ; q^{2}\right)_{n}$, and using that $n(n+1)=m(m+1)$, this yields

$$
\sum_{r=m}^{\infty} \frac{(q ; q)_{2 r+1} q^{r}}{(-q ; q)_{2 r+2}\left(q^{2} ; q^{2}\right)_{r-m}\left(q^{2} ; q^{2}\right)_{r+m+1}}=q^{-m(m+1)} \sum_{r=m}^{\infty} q^{r(r+2)} .
$$


After the usual shift in summation indices this may be recognized as the $a=q^{2 m+2}$ instance of 3.3 .

Theorem 4. If we take $u_{n}=v_{n}=1 /\left(q^{2} ; q^{2}\right)_{n}$ and

$$
\delta_{n}=(q ; q)_{2 n+1} q^{n}
$$

in the Asymmetric Bilateral Bailey Transform, then

$$
\gamma_{n}=\sum_{r=0}^{\infty} q^{r(r+3) / 2+(2 r+1) m}=q^{-2 n(n+1)} \sum_{r=2 m}^{\infty} q^{r(r+3) / 2},
$$

where $m=\max \{n,-n-1\}$.

Proof. Substituting (3.8) and $u_{n}=1 /\left(q^{2} ; q^{2}\right)_{n}$ into (3.7) and again using that $n(n+1)=m(m+1)$ yields

$$
\sum_{r=m}^{\infty} \frac{(q ; q)_{2 r+1} q^{r}}{\left(q^{2} ; q^{2}\right)_{r-m}\left(q^{2} ; q^{2}\right)_{r+m+1}}=q^{-2 m(m+1)} \sum_{r=2 m}^{\infty} q^{r(r+3) / 2} .
$$

By a shift in the summation indices this may be recognized as the $a=q^{2 m+2}$ instance of (3.4).

\section{Ramanujan's False Theta Identities}

Theorem 1 now allows us to give uniform proofs of $1.1 \mathrm{a}-1.1 \mathrm{~d}$ ). Namely, by combining the Symmetric Bilateral Bailey Transform with Theorem 1 , we obtain

Theorem 5. If for $n$ a nonnegative integer

$$
\beta_{n}=\sum_{r=-n}^{n} \frac{\alpha_{n}}{\left(q^{2} ; q^{2}\right)_{n-r}\left(q^{2} ; q^{2}\right)_{n+r}}
$$

then

$$
\sum_{n=0}^{\infty} \frac{\left(q^{2} ; q^{2}\right)_{2 n} q^{n} \beta_{n}}{(-q ; q)_{2 n+1}}=\sum_{j=0}^{\infty} q^{j(j+1)} \sum_{n=-j}^{j} \alpha_{n} q^{-n^{2}}
$$

subject to conditions on $\alpha_{n}$ and $\beta_{n}$ that make the series absolutely convergent.

Proof. We use the conjugate Bailey pair $\left(\gamma_{n}, \delta_{n}\right)$ of Theorem 1 in the Symmetric Bilateral Bailey Transform with $u_{n}=v_{n}=1 /\left(q^{2} ; q^{2}\right)_{n}$. Hence

$$
\begin{aligned}
\sum_{n=0}^{\infty} \frac{\left(q^{2} ; q^{2}\right)_{2 n} q^{n} \beta_{n}}{(-q ; q)_{2 n+1}} & =\sum_{n=0}^{\infty} \beta_{n} \delta_{n} \\
& =\sum_{n=-\infty}^{\infty} \alpha_{n} \gamma_{n} \\
& =\sum_{n=-\infty}^{\infty} \alpha_{n} q^{-n^{2}} \sum_{j=|n|}^{\infty} q^{j(j+1)} \\
& =\sum_{j=0}^{\infty} q^{j(j+1)} \sum_{n=-j}^{j} \alpha_{n} q^{-n^{2}}
\end{aligned}
$$


Before proceeding we remark that the Bailey pair (4.1) may readily be related to the more standard definition of such pairs 2, 3, 17. Specifically, if we define $\bar{\alpha}_{0}=\alpha_{0}$ and, for $n>0, \bar{\alpha}_{n}=\alpha_{n}+\alpha_{-n}$, then $\left(\bar{\alpha}_{n}, \beta_{n}\right)$ is a Bailey pair with $a=1$ and $q \rightarrow q^{2}$. From this comment it follows that we may utilize L. J. Slater's compendium of Bailey pairs [14, as well as other sources [5, 2], to obtain false theta identities.

Theorem 6. Identity 1.1a is valid, i.e.,

$$
\sum_{n=0}^{\infty} \frac{(-1)^{n} q^{n(n+1)}\left(q ; q^{2}\right)_{n}}{(-q ; q)_{2 n+1}}=\sum_{n=0}^{\infty}(-1)^{n} q^{n(n+1) / 2}
$$

Proof. We apply Theorem 5 with [14, G(4)]

$$
\alpha_{n}=(-1)^{n} q^{n(n+1) / 2} \quad \text { and } \quad \beta_{n}=\frac{(-1)^{n} q^{n^{2}}\left(q ; q^{2}\right)_{n}}{\left(q^{2} ; q^{2}\right)_{2 n}} .
$$

Thus

$$
\begin{aligned}
\sum_{n=0}^{\infty} \frac{(-1)^{n} q^{n(n+1)}\left(q ; q^{2}\right)_{n}}{(-q ; q)_{2 n+1}} & =\sum_{n=0}^{\infty} \frac{\left(q^{2} ; q^{2}\right)_{2 n} q^{n} \beta_{n}}{(-q ; q)_{2 n+1}} \\
& =\sum_{j=0}^{\infty} q^{j(j+1)} \sum_{n=-j}^{j} \alpha_{n} q^{-n^{2}} \\
& =\sum_{j=0}^{\infty} q^{j(j+1)} \sum_{n=-j}^{j}(-1)^{n} q^{-\left(\begin{array}{c}
n \\
2
\end{array}\right)}
\end{aligned}
$$

It is an easy exercise in mathematical induction on $j$ to show that

$$
\sum_{n=-j}^{j}(-1)^{n} q^{-\left(\begin{array}{c}
n \\
2
\end{array}\right)}=(-1)^{j} q^{-\left(\begin{array}{c}
j+1 \\
2
\end{array}\right)}
$$

and 4.3 follows.

Theorem 7. Identities (1.1b and 1.4 are valid.

Proof. Clearly (1.1b) is the special case $z=-1$ of 1.4 . Furthermore with

$$
\alpha_{n}=z^{n} q^{n^{2}}
$$

in 4.1), we see that

$$
\beta_{n}=\sum_{r=-n}^{n} \frac{z^{n} q^{n^{2}}}{\left(q^{2} ; q^{2}\right)_{n-r}\left(q^{2} ; q^{2}\right)_{n+r}}=\frac{\left(-z q ; q^{2}\right)_{n}\left(-z^{-1} q ; q^{2}\right)_{n}}{\left(q^{2} ; q^{2}\right)_{2 n}}
$$


by [1, p. 49, Ex. 1]. Consequently, by Theorem 5

$$
\begin{aligned}
\sum_{n=0}^{\infty} \frac{\left(-z q ; q^{2}\right)_{r}\left(-z^{-1} q ; q^{2}\right)_{n} q^{n}}{(-q ; q)_{2 n+1}} & =\sum_{n=0}^{\infty} \frac{\left(q^{2} ; q^{2}\right)_{2 n} q^{n} \beta_{n}}{(-q ; q)_{2 n+1}} \\
& =\sum_{j=0}^{\infty} q^{j(j+1)} \sum_{n=-j}^{j} \alpha_{n} q^{-n^{2}} \\
& =\sum_{j=0}^{\infty} q^{j(j+1)} \sum_{n=-j}^{j} z^{n} \\
& =\sum_{j=0}^{\infty} \frac{1-z^{2 j+1}}{1-z} z^{-j} q^{j(j+1)}
\end{aligned}
$$

In passing we note that for $z=1$ the identity (1.4) simplifies to

$$
\sum_{n=0}^{\infty} \frac{\left(-q ; q^{2}\right)_{n} q^{n}}{\left(-q^{2} ; q^{2}\right)_{n}\left(1+q^{2 n+1}\right)}=\sum_{n=0}^{\infty}(2 n+1) q^{n(n+1)}
$$

which is similar to (1.6). Also, for $z=q^{2}$, the sum on the right of 1.4 telescopes and we obtain

$$
(1-q) \sum_{n=0}^{\infty} \frac{\left(-q ; q^{2}\right)_{n} q^{n}}{\left(-q^{2} ; q^{2}\right)_{n+1}}=1 .
$$

Theorem 8. Identity 1.1c is valid.

Proof. We apply Theorem 5 with [14, G(1)]

$$
\alpha_{n}=(-1)^{n} q^{n(3 n+1) / 2} \quad \text { and } \quad \beta_{n}=\frac{\left(q ; q^{2}\right)_{n}}{\left(q^{2} ; q^{2}\right)_{2 n}} .
$$

Thus

$$
\begin{aligned}
\sum_{n=0}^{\infty} \frac{\left(q ; q^{2}\right)_{n} q^{n}}{(-q ; q)_{2 n+1}} & =\sum_{n=0}^{\infty} \frac{\left(q^{2} ; q^{2}\right)_{2 n} q^{n} \beta_{n}}{(-q ; q)_{2 n+1}} \\
& =\sum_{j=0}^{\infty} q^{j(j+1)} \sum_{n=-j}^{j} \alpha_{n} q^{-n^{2}} \\
& =\sum_{j=0}^{\infty} q^{j(j+1)} \sum_{n=-j}^{j}(-1)^{n} q^{\left(\begin{array}{c}
n+1 \\
2
\end{array}\right)} \\
& =\sum_{j=0}^{\infty}(-1)^{j} q^{3 j(j+1) / 2},
\end{aligned}
$$

by (4.4) with $q$ replaced by $1 / q$.

Theorem 9. Identity $1.1 \mathrm{~d}$ is valid.

Proof. We apply Theorem 5 with [14, H(2)]

$$
\alpha_{n}=(-1)^{n} q^{n(2 n+1)} \quad \text { and } \quad \beta_{n}=\frac{1}{(-q ;-q)_{2 n}} .
$$


So

$$
\begin{aligned}
\sum_{n=0}^{\infty} \frac{(q ;-q)_{2 n} q^{n}}{(-q ; q)_{2 n+1}} & =\sum_{n=0}^{\infty} \frac{\left(q^{2} ; q^{2}\right)_{2 n} q^{n} \beta_{n}}{(-q ; q)_{2 n+1}} \\
& =\sum_{j=0}^{\infty} q^{j(j+1)} \sum_{n=-j}^{j} \alpha_{n} q^{-n^{2}} \\
& =\sum_{j=0}^{\infty} q^{j(j+1)} \sum_{n=-j}^{j}(-1)^{n} q^{n(n+1)} \\
& =\sum_{j=0}^{\infty}(-1)^{j} q^{2 j(j+1)}
\end{aligned}
$$

by (4.4) with $q$ replaced by $1 / q^{2}$.

Identity $1.1 \mathrm{e}$ apparently does not follow as easily from Theorems 14.

\section{Further APPLiCATions}

Each of the Theorems 24 can be combined with the relevant Bailey transform to produce analogs of Theorem 5. In each case the proof is a mirror image of that given for Theorem 5 , and is consequently omitted.

Theorem 10. If for $n$ a nonnegative integer

$$
\beta_{n}=\sum_{r=-n}^{n} \frac{\alpha_{n}}{\left(q^{2} ; q^{2}\right)_{n-r}\left(q^{2} ; q^{2}\right)_{n+r}},
$$

then

$$
\sum_{n=0}^{\infty}(q ; q)_{2 n} q^{n} \beta_{n}=\sum_{j=0}^{\infty} q^{\left(\begin{array}{c}
j+1 \\
2
\end{array}\right)} \sum_{n=-\left\lfloor\frac{j}{2}\right\rfloor}^{\left\lfloor\frac{j}{2}\right\rfloor} \alpha_{n} q^{-2 n^{2}},
$$

subject to the usual absolute convergent conditions.

Theorem 11. If for $n$ a nonnegative integer

$$
\beta_{n}=\sum_{r=-n-1}^{n} \frac{\alpha_{r}}{\left(q^{2} ; q^{2}\right)_{n-r}\left(q^{2} ; q^{2}\right)_{n+r+1}},
$$

then

$$
\sum_{n=0}^{\infty} \frac{\left(q^{2} ; q^{2}\right)_{2 n+1} q^{n} \beta_{n}}{(-q ; q)_{2 n+2}}=\sum_{j=0}^{\infty} q^{j(j+2)} \sum_{n=-j-1}^{j} \alpha_{n} q^{-n(n+1)}
$$

and

$$
\sum_{n=0}^{\infty}(q ; q)_{2 n+1} q^{n} \beta_{n}=\sum_{j=0}^{\infty} q^{j(j+3) / 2} \sum_{n=-\left\lfloor\frac{j}{2}\right\rfloor-1}^{\left\lfloor\left\lfloor\frac{j}{2}\right\rfloor\right.} \alpha_{n} q^{-2 n(n+1)},
$$

subject to the usual absolute convergent conditions. 
The number of possible further applications of Theorems 5,10 and 11 is huge. Indeed each of these theorems will take a Bailey pair and produce a new identity. In light of the enormous number of known Bailey pairs (c.f. [2, [5]), we will only provide a minimal sample in this section.

Our first example is one of the elegant formulas from Ramanujan's Lost Notebook [4. p. 239, Entry 9.5.3].

Theorem 12. Identity 1.5 is valid.

Proof. In Theorem 5 we use the Bailey pair [14, C(1)]

$$
\begin{gathered}
\alpha_{2 m}=(-1)^{m} q^{2 m(3 m+1)}, \quad \alpha_{2 m+1}=0 \\
\beta_{n}=\frac{1}{\left(q^{2} ; q^{2}\right)_{n}\left(q^{2} ; q^{4}\right)_{n}} .
\end{gathered}
$$

Then

$$
\begin{aligned}
\sum_{n=0}^{\infty} \frac{q^{n}}{\left(-q ; q^{2}\right)_{n+1}} & =\sum_{j=0}^{\infty} q^{j(j+1)} \sum_{m=-\left\lfloor\frac{j}{2}\right\rfloor}^{\left\lfloor\frac{j}{2}\right\rfloor} \alpha_{2 m} q^{-4 m^{2}} \\
& =\sum_{j=0}^{\infty} q^{j(j+1)} \sum_{m=-\left\lfloor\frac{j}{2}\right\rfloor}^{\left\lfloor\frac{j}{2}\right\rfloor}(-1)^{m} q^{2 m(m+1)} \\
& =\sum_{j=0}^{\infty}(-1)^{\left\lfloor\frac{j}{2}\right\rfloor} q^{j(j+1)+2\left\lfloor\frac{j}{2}\right\rfloor\left(\left\lfloor\frac{j}{2}\right\rfloor+1\right)} \\
& =\sum_{j=0}^{\infty}(-1)^{j} q^{2 j(3 j+2)}\left(1+q^{4 j+2}\right) .
\end{aligned}
$$

As the next result reveals, there is no need to restrict ourselves to single fold series.

Theorem 13. We have

$$
\sum_{n, j=0}^{\infty} \frac{q^{j(2 j+1)+n}\left(q^{2} ; q^{2}\right)_{n+j}}{(-q ; q)_{2 n+2 j+1}\left(q^{2} ; q^{2}\right)_{j}\left(q^{2} ; q^{2}\right)_{n}}=\sum_{n=0}^{\infty}(-1)^{n} q^{n(5 n+3)}\left(1+q^{4 n+2}\right)
$$

Proof. In Theorem 5 we require Bressoud's Bailey pair [2, p. 280, Eqs. (5.6) and (5.7) with $\left.a=1, q \rightarrow q^{2}\right]$ :

$$
\begin{aligned}
& \alpha_{2 m}=(-1)^{m} q^{m(5 m+1)}, \quad \alpha_{2 m+1}=0 \\
& \beta_{n}=\frac{1}{\left(q^{2} ; q^{2}\right)_{2 n}} \sum_{j=0}^{n} \frac{\left(q^{2} ; q^{2}\right)_{n} q^{2 j^{2}}}{\left(q^{2} ; q^{2}\right)_{j}\left(q^{2} ; q^{2}\right)_{n-j}}
\end{aligned}
$$


Then

$$
\begin{aligned}
\sum_{n=0}^{\infty} \frac{q^{n}}{(-q ; q)_{2 n+1}} & \sum_{j=0}^{n} \frac{\left(q^{2} ; q^{2}\right)_{n} q^{2 j^{2}}}{\left(q^{2} ; q^{2}\right)_{j}\left(q^{2} ; q^{2}\right)_{n-j}} \\
& =\sum_{j=0}^{\infty} q^{j(j+1)} \sum_{m=-\left\lfloor\frac{j}{2}\right\rfloor}^{\left\lfloor\frac{j}{2}\right\rfloor} \alpha_{2 m} q^{-4 m^{2}} \\
& =\sum_{j=0}^{\infty} q^{j(j+1)} \sum_{m=-\left\lfloor\frac{j}{2}\right\rfloor}^{\left\lfloor\frac{j}{2}\right\rfloor}(-1)^{m} q^{m(m+1)} \\
& =\sum_{j=0}^{\infty}(-1)^{\left\lfloor\frac{j}{2}\right\rfloor} q^{j(j+1)+\left\lfloor\frac{j}{2}\right\rfloor\left(\left\lfloor\frac{j}{2}\right\rfloor+1\right)} \\
& =\sum_{j=0}^{\infty}(-1)^{j} q^{j(5 j+3)}\left(1+q^{4 j+2}\right) .
\end{aligned}
$$

Changing the order of the sums on the left and changing $n \rightarrow n+j$ completes the proof.

As our second-last example we prove identity (1.7).

Theorem 14. Identity (1.7) is true.

Proof. We recall the Bailey pair [15, I(14)]

$$
\begin{gathered}
\alpha_{2 m}=(-1)^{m} q^{2 m(2 m+1)}, \quad \alpha_{2 m+1}=0 \\
\beta_{n}=\frac{\left(-q^{4} ; q^{4}\right)_{n-1}\left(-q^{2} ; q^{2}\right)_{n}}{\left(-q^{2}, n-1, q^{2}\right)\left(q^{2} ; q^{2}\right)_{2 n}} .
\end{gathered}
$$

Following the previous two proofs we get

$$
\sum_{n=0}^{\infty} \frac{\left(-q^{4} ; q^{4}\right)_{n-1} q^{n}}{\left(-q^{2} ; q^{2}\right)_{n-1}\left(-q ; q^{2}\right)_{n+1}}=\sum_{j=0}^{\infty} q^{j(j+1)} \sum_{m=-\left\lfloor\frac{j}{2}\right\rfloor}^{\left\lfloor\frac{j}{2}\right\rfloor}(-1)^{m} q^{2 m} .
$$

One can simply perform the sum over $m$ leading to

$$
\sum_{n=0}^{\infty} \frac{\left(-q^{4} ; q^{4}\right)_{n-1} q^{n}}{\left(-q^{2} ; q^{2}\right)_{n-1}\left(-q ; q^{2}\right)_{n+1}}=\frac{1}{1+q^{2}}\left(1+2 q \sum_{n=0}^{\infty}(-1)^{n} q^{4 n(n+1)}\right),
$$

but this does not capture the strange expansion of the series under consideration.

Instead we draw a little table of the first few terms occurring on the right-hand side of 5.3. Each row corresponds to a fixed $j$-value $(0,1,2,3,4)$ and has $m$ ranging from $-\left\lfloor\frac{j}{2}\right\rfloor$ to $\left\lfloor\frac{j}{2}\right\rfloor$ :

$$
\begin{aligned}
& 1 \\
& \begin{array}{lll} 
& q^{2} & \\
-q^{4} & q^{6} & -q^{8}
\end{array} \\
& -q^{10} \quad q^{12}-q^{14} \\
& q^{16}-q^{18} q^{20}-q^{22} \quad q^{24} \text {. }
\end{aligned}
$$


Once the pattern has been observed the proof is elementary. Writing the summand on the right of 5.3 as $(-1)^{m} q^{2 E_{j, m}}$ (so that $E_{j, m}=j(j+1) / 2+m$ ), it is readily checked that

$$
E_{j+1,-\left\lfloor\frac{j}{2}\right\rfloor}=E_{j,\left\lfloor\frac{j}{2}\right\rfloor}+1 .
$$

This shows that all even powers of $q$ occur on the right of (5.3). It only remains to establish the sign of an arbitrary term $q^{2 n}$ in the series. To determine this, note that consecutive terms have opposite sign except the terms corresponding to $E_{2 j, j}$ and $E_{2 j+1,-j}$. We thus get

$$
1+\sum_{n=1}^{\infty}(-1)^{f(n)} q^{2 n}
$$

with

$$
f(n)=n+j \quad \text { if } \quad E_{2 j-1,1-j} \leq n \leq E_{2 j, j} .
$$

So far all in all our applications we have employed Theorem 5, and in our last example we explore one of the many applications of Theorem 11.

Theorem 15. We have

$$
\begin{aligned}
\sum_{n=0}^{\infty} \frac{\left(-z q^{2} ; q^{2}\right)_{n}\left(-z^{-1} q^{2} ; q^{2}\right)_{n} q^{n}}{(-q ; q)_{2 n+2}} & =\sum_{n=0}^{\infty} \frac{1-z^{2 n+2}}{1-z^{2}} z^{-n} q^{n(n+2)} \\
& =\sum_{n=0}^{\infty} \frac{1+z^{2}+z^{4}+\cdots+z^{2 n}}{z^{n}} q^{n(n+2)}
\end{aligned}
$$

For $z=1$ this give rise to 1.6 and for $z=-1$ to

$$
\sum_{n=1}^{\infty} \frac{\left(q^{2} ; q^{2}\right)_{n-1}^{2} q^{n}}{(-q ; q)_{2 n}}=\sum_{n=1}^{\infty} n(-1)^{n-1} q^{n^{2}} .
$$

Proof. From the $q$-binomial theorem [8, Equation (II.4)]

$$
{ }_{1} \phi_{0}\left(q^{-n} ;-; q, z\right)=\left(z q^{-n} ; q\right)_{n}
$$

one may deduce that the following companion to the Bailey pair 4.5 satisfies equation (5.1):

$$
\alpha_{n}=z^{n} q^{n^{2}}, \quad \beta_{n}=\frac{\left(-z q ; q^{2}\right)_{n}\left(-z^{-1} q ; q^{2}\right)_{n+1}}{\left(q^{2} ; q^{2}\right)_{2 n+1}} .
$$

Substituting this in $(5.2)$ and replacing $z \rightarrow z q$, we obtain

$$
\sum_{n=0}^{\infty} \frac{\left(-z q^{2} ; q^{2}\right)_{n}\left(-z^{-1} ; q^{2}\right)_{n+1} q^{n}}{(-q ; q)_{2 n+2}}=\sum_{j=0}^{\infty} \frac{1-z^{2 j+2}}{1-z} z^{-j-1} q^{j(j+2)} .
$$

Dividing both sides by $\left(1+z^{-1}\right)$ gives the desired result.

\section{Conclusion}

This paper provides a new direction for the Bailey transform. In works such as [2], the emphasis has been on picking up new Bailey pairs $\left(\alpha_{n}, \beta_{n}\right)$, and the conjugate Bailey pairs $\left(\gamma_{n}, \delta_{n}\right)$ have been restricted to one specific instance. There have been several investigations of alternatives to the standard conjugate Bailey pairs (see e.g., 6, 13, 11, 12); however none seem to capture the type of results given in $1.1 \mathrm{a}-1.1 \mathrm{~d}$. 
In this initial work, we have restricted ourselves to false theta series applications. However, the Hecke type series involving indefinite quadratic forms clearly fall within the purview of these methods and will be dealt with in a subsequent investigation.

\section{REFERENCES}

[1] G. E. Andrews, The Theory of Partitions, Encycl. of Math. and Its Appl., Vol. 2, AddisonWesley, Reading, 1976. (Reissued: Cambridge University Press, 1985) 4

[2] G. E. Andrews, Multiple series Rogers-Ramanujan type identities, Pacific J. Math. 114 (1984), 267-283. 4, 5,6

[3] G. E. Andrews, Bailey's transform, lemma, chains and tree, in Special Functions 2000: Current Perspective and Future Directions, pp. 1-22, J. Bustoz et al. eds., Kluwer Academic Publishers, Dordrecht, 2001. 4

[4] G. E. Andrews and B. C. Berndt, Ramanujan's Lost Notebook, Vol. 1, Springer, New York, 2005. 110 5

[5] W. N. Bailey, Identities of the Rogers-Ramanujan type, Proc. London Math. Soc. (2) 50 (1949), 1-10. 24,5

[6] D. M. Bressoud, Some identities for terminating q-series, Math. Proc. Camb. Phil. Soc. 89 (1981), 211-223. 6

[7] N. J. Fine, Basic Hypergeometric Series and Applications, Mathematical Surveys and Monographs, Vol. 27, AMS, Providence, Rhode Island, 1888. 3

[8] G. Gasper and M. Rahman, Basic Hypergeometric Series, 2nd ed., Encycl. of Math and Its Appl., Vol. 96, Cambridge University Press, Cambridge, 2004. 3 , 3

[9] S. Ramanujan, The Lost Notebook and Other Unpublished Papers, Narosa, New Delhi, 1988. 1

[10] L. J. Rogers, On two theorems of combinatory analysis and some allied identities, Proc. London Math. Soc. (2) 16 (1917), 315-336. 1

[11] A. Schilling and S. O. Warnaar, A higher level Bailey lemma: proof and application, Ramanujan J. 2 (1998), 327-349. 6

[12] A. Schilling and S. O. Warnaar, Conjugate Bailey pairs. From configuration sums and fractional-level string functions to Bailey's lemma, Contemp. Math. 297 (2002), 227-255. 6

[13] U. B. Singh, A note on a transformation of Bailey, Quart. J. Math. Oxford Ser. (2) 45 (1994), 111-116. 6

[14] L. J. Slater, A new proof of Rogers' transformations of infinite series, Proc. London Math. Soc. (2) 53 (1951), 460-475. $4,4,4,5$

[15] L. J. Slater, Further identities of the Rogers-Ramanujan type, Proc. London Math. Soc. (2) 54 (1952), 147-167. 5

[16] L. J. Slater, Generalized Hypergeometric Functions, Cambridge University Press, Cambridge, 1966. 2

[17] S. O. Warnaar, 50 Years of Bailey's lemma, in Algebraic Combinatorics and Applications, pp. 333-347, A. Betten et al. eds., Springer, Berlin, 2001.4

The Pennsylvania State University, University Park, PA 16802

Department of Mathematics and Statistics, The University of Melbourne, ViC 3010, Australia 